

\title{
Elemental and isotopic composition measurements of galactic cosmic rays
}

M. E. Wiedenbeck

Citation: AIP Conference Proceedings 1516, 150 (2013); doi: 10.1063/1.4792559

View online: http://dx.doi.org/10.1063/1.4792559

View Table of Contents: http://scitation.aip.org/content/aip/proceeding/aipcp/1516?ver=pdfcov

Published by the AIP Publishing

Articles you may be interested in

Cosmic ray source abundances and the acceleration of cosmic rays

AIP Conf. Proc. 528, 437 (2000); 10.1063/1.1324355

Cosmic ray acceleration in superbubbles and the composition of cosmic rays

AIP Conf. Proc. 528, 375 (2000); 10.1063/1.1324342

Cosmic-Ray Isotopic Composition results from the ACE mission

AIP Conf. Proc. 516, 301 (2000); 10.1063/1.1291484

Ultra heavy ( $Z>30)$ and heavy (10 Z 30) cosmic-ray measurements on ACCESS with the charge $(Z)$ identification module (ZIM)

AIP Conf. Proc. 458, 251 (1999); 10.1063/1.57688

The international space station as an observatory for cosmic-ray physics and astrophysics

AIP Conf. Proc. 458, 133 (1999); 10.1063/1.57682 


\title{
Elemental and Isotopic Composition Measurements of Galactic Cosmic Rays
}

\author{
M. E. Wiedenbeck \\ Jet Propulsion Laboratory, California Institute of Technology \\ M.S. 169-327, 4800 Oak Grove Dr., Pasadena, CA 91109 USA
}

\begin{abstract}
Measurements of the relative abundances of elements and their isotopes in galactic cosmic rays, which include both stable and long lived radioactive species, have led to a variety of insights into the nature of the cosmic ray source, the mechanisms involved in accelerating particles to high energies, and characteristics of their transport in the Galaxy. Some key developments that have contributed to the present understanding of cosmic rays are summarized.
\end{abstract}

Keywords: cosmic rays, composition, cosmic-ray isotopes, propagation, acceleration

PACS: $26.40 .+\mathrm{r}, 95.55 . \mathrm{Vj}$, 95.85.Ry, 98.70.Sa

\section{INTRODUCTION.}

The observation in 1948 of tracks produced by highly ionizing penetrating particles in balloon-borne nuclear emulsions [1] demonstrated that galactic cosmic rays (GCRs) contain elements heavier than $\mathrm{H}$ and He. Historical accounts of this discovery are given in $[2,3]$. Work carried out over the next two decades demonstrated that essentially all the elements in the periodic table are represented in cosmic rays. While these heavy nuclei constitute no more than a few percent of the GCRs by number, they have proven to be an invaluable source of information for understanding the source material, the acceleration, and the galactic propagation of the cosmic rays. This paper briefly summarizes some of the key advances that have been made as the result of elemental and isotopic composition studies, emphasizing composition up through the iron group (atomic numbers $Z \lesssim 30$ ). The "ultraheavy" elements $(Z \gtrsim 30)$ are discussed elsewhere in this volume $[4,5]$.

By the 1960s nuclear emulsions had largely been replaced by detector systems with electronic readout as the sensors of choice for GCR composition studies. At the time of the solar minimum between sunspot cycles 19 and $20(\sim 1964-66)$, small detector systems for studying galactic cosmic rays had been flown on satellites and larger instruments were regularly being carried high in the atmosphere by balloons. Typically two or more sensor elements operated in coincidence provided signals dependent on the nuclear charge, $Z$, and the energy per nucleon, $E / M$, of detected particles. With appropriate choices of the sensors, the identity and energy of the particles could be determined allowing GCR composition and energy spectra to be derived. The status of the field in the late 1960s was reviewed in [6]. The subsequent exposure of a large, optimized sensor system on the Earth-orbiting HEAO-3 satellite [7] set a new standard for precise elemental composition measurements at $\mathrm{GeV} /$ nuc energies [8].

In many cases the processes that form or subsequently modify abundances differ among the isotopes of an element. However, as illustrated in Figure 1, the instrumental 
precision required to separate isotopes significantly exceeds that needed for identifying elements. By about 1970 much of the focus of new instrumentation for cosmic ray composition studies for elements up through the iron group had turned to achieving the measurement precision required for isotope studies. Of particular importance was the addition of "hodoscopes", sensors capable of measuring the trajectories of the detected cosmic rays so that corrections could be made for particle pathlengths through the other detectors $[9,10]$. This innovation, which became possible because of miniaturization of electronics, is now routinely used in the designs of composition instruments $[11,12]$.

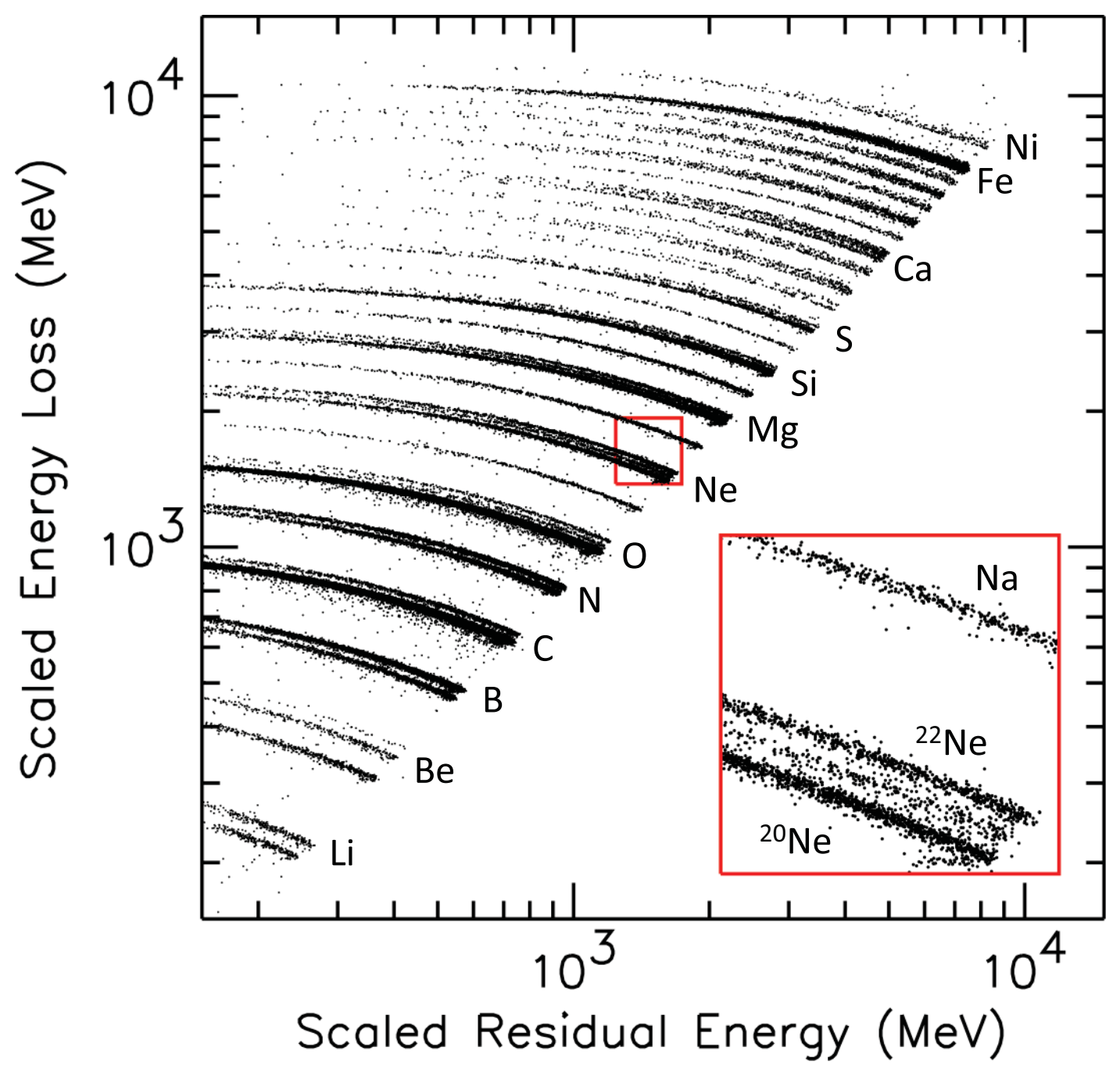

FIGURE 1. Sample of flight data from the Cosmic Ray Isotope Spectrometer [12] on the Advanced Composition Explorer satellite. Energy loss and residual energy measured in silicon solid state detectors have been corrected for particle angle of incidence obtained from the instrument's scintillating optical fiber hodoscope. Events due to individual elements appear as distinct tracks that are made of up of subtracks caused by the isotopes of the element (inset). 


\section{COMPOSITION HIGHLIGHTS}

In the following paragraphs we briefly summarize a number of major advances in the understanding of the galactic cosmic rays that have come about through studies of the elemental and isotopic composition of this material. Due to space limitations only selected references are given, with emphasis on some of the earliest papers on each topic together with works that were particularly influential or that reflect the current status.

Secondary Elements. Elements having low abundances relative to heavier species in solar system matter have much higher relative abundances in cosmic rays. Nuclear collisions during the transport of the high energy particles through the Galaxy cause a fraction of the nuclei to fragment into lighter particles, which are referred to as "secondary" cosmic rays to distinguish them from the "primary" nuclei synthesized in stars. Secondary elements include $\mathrm{Li}, \mathrm{Be}$, and $\mathrm{B}(3 \leq Z \leq 5)$, elements in the subFe region $(21 \leq Z \lesssim 25)$, and a number of odd- $Z$ elements. Some elements (e.g., N) contain significant fractions of both secondary and primary material. The amount of matter traversed in the Galaxy (i.e., the thickness of the target causing the fragmentation) can be inferred from the abundances of dominantly secondary species. From early measurements using emulsions it was established that this target thickness was not great enough to erase the imprint of stellar nucleosynthesis processes on the abundances of the major cosmic ray elements [13]. Reviews of the extensive literature on GCR secondaries include $[14,15,16]$ and recent results are presented in $[17,18]$.

Secondary Radionuclides. Nuclear fragmentation produces both stable and radioactive secondaries. The radioactive nuclides that beta decay with halflives much shorter than the time that cosmic rays spend in the Galaxy just contribute to the abundance of first stable nuclide that occurs in their decay chain. However, for nuclides that can decay only by orbital electron capture the decays are prevented by the absence of orbital electrons, causing these species to become effectively stable at cosmic ray energies. For example, ${ }^{7} \mathrm{Be}$ (laboratory halflife of 53 days) is the most abundant Be isotope in the arriving cosmic rays. Nuclides with beta-decay halflives comparable to the time that cosmic rays spend in the Galaxy are used as clocks to determine that residence time. The most important example is ${ }^{10} \mathrm{Be}$, which undergoes $\beta^{-}$decay with a halflife of $1.4 \mathrm{Myr}$. This nuclide has been used to infer that cosmic rays spend $\sim 10-20$ Myr in the Galaxy (a value that is somewhat dependent on the cosmic ray transport model adopted). The combination of the residence time and the amount of matter traversed results in an estimate of the mean density sampled by the cosmic rays and results in a relatively low value that implies that a significant fraction of cosmic ray propagation occurs in the low-density halo of the Galaxy. The derivation of the time scale for cosmic ray propagation using measurements of ${ }^{10} \mathrm{Be}$ have been discussed in $[14,19,20]$ and recent results including other clock isotopes are given by $[21,22,16]$ and references therein.

Similarities to Solar System Abundances. The most prominent elements observed in the galactic cosmic rays are the same as in solar system material and in many other samples of matter: $\mathrm{H}, \mathrm{He}, \mathrm{C}, \mathrm{O}, \mathrm{Ne}, \mathrm{Mg}, \mathrm{Si}$, and Fe. The heavy nuclides in this list are also the major products of stellar nucleosynthesis. After taking elemental fractionation effects into account (see below), the relative abundances in cosmic ray source material and in the solar system are remarkably similar $[8,23]$. One of the early motivations for 
studying the isotopic composition of cosmic rays was the hope that the source material might bear compositional signatures associated with a particular nucleosynthesis process and thus help in identifying a class of stellar objects responsible for the origin and acceleration of GCRs. However, the observations showed that the cosmic ray source contains contributions from a wide range of nucleosynthesis processes (as does the solar system) and provided a strong indication that cosmic rays are an accelerated sample of interstellar matter [24, 25]. Thus recent work has concentrated on searching for differences between GCR source and solar system composition that could probe the physical conditions peculiar to the origin of these two samples of interstellar material.

Elemental Fractionation. Composition comparisons between cosmic ray source material (as derived by correcting abundances measured in the arriving cosmic rays for secondary contributions and for losses during galactic propagation) and solar system matter show differences up to a factor $\sim 4-5$. A key step in understanding cosmic ray composition came with the realization that these differences are ordered by atomic properties of the elements $[26,27]$. Although originally parameterized in terms of first ionization potential (FIP), it was recognized that FIP is closely correlated with the volatility of the elements and thus the physical mechanism for the fractionation could depend on the relative amounts of the various element that are present in grains rather than in the gas phase in the medium from which cosmic rays are accelerated. An essential contribution to these ideas was the realization that charged grains, which have high magnetic rigidity, would be accelerated by supernova shocks, with subsequent sputtering producing suprathermal ions that could be further accelerated with high efficiency [28, 29, 30, 31].

Isotopic Anomalies. Measurements of isotopic composition showed, in most cases, that nuclidic abundances in cosmic ray source material closely resemble those found in the solar system [24, 21]. One striking exception was found: the abundance ratio ${ }^{22} \mathrm{Ne} /{ }^{20} \mathrm{Ne}$ is strongly enhanced $[32,33,34]$, exceeding the solar wind value by a factor $\sim 5$. It was noted that Wolf-Rayet (WR) stars, massive stars in a phase where their outer $\mathrm{H}$ envelope has been ejected in a stellar wind, have surface abundances dominated by the products of nuclear reactions that occur during He burning and that these products should have a large overabundance of ${ }^{22} \mathrm{Ne}$. Thus the cosmic ray excess of ${ }^{22} \mathrm{Ne}$ could be attributable to a significant contribution of WR star ejecta to the seed material accelerated by interstellar shocks [35]. Model calculations suggested that the isotopic compositions of most other elements should be minimally affected by the WR contribution required to explain the ${ }^{22} \mathrm{Ne}$, with ${ }^{58} \mathrm{Fe} /{ }^{56} \mathrm{Fe}$ being the isotope ratio expected to show the next largest effect [36]. A possible excess of ${ }^{58} \mathrm{Fe}$ was noted as was an enhancement of ${ }^{12} \mathrm{C} /{ }^{16} \mathrm{O}$ that could be attributable to an admixture of WR ejecta in the GCR source material [37].

Primary Radionuclides. As noted above, radionuclides that decay only by orbital electron capture (ec) are effectively stable at cosmic ray energies. This effect was suggested as a basis for determining the time between synthesis and ejection of such nuclides in a supernova explosion and their subsequent acceleration to cosmic ray energies [38]. Measurements of the isotopic composition of $\mathrm{Ni}$ showed that one such ec nuclide, ${ }^{59} \mathrm{Ni}$ (halflife $\sim 10^{5} \mathrm{yr}$ ), is essentially absent in the cosmic rays while its daughter product ${ }^{59} \mathrm{Co}$ clearly includes a primary component, some of which could have come from ${ }^{59} \mathrm{Ni}$ decay [40]. Thus it was concluded that the material that gets accelerated 
to cosmic ray energies typically resides in the interstellar medium for $>10^{5} \mathrm{yr}$ prior to acceleration and that the bulk of the cosmic rays are not produced by supernovae accelerating their own nucleosynthesis products.

Cosmic Ray Modulation. In the energy range below a few $\mathrm{GeV} /$ nuc, where composition measurements are the most precise, cosmic-ray energy spectra observed near Earth have been altered by the particles' interaction with the magnetic field carried by the expanding solar wind. This "solar modulation" should lead to an adiabatic energy loss amounting to several hundred $\mathrm{MeV} /$ nuc. It was suggested that a direct measure of the magnitude of this energy loss could be obtained using observations of the energy spectra of secondary electron capture (ec) nuclides having short laboratory halflives. At sufficiently low energies these nuclei have a significant probability of attaching an electron from an atom of the interstellar gas and then undergoing ec decay. At higher energy the attachment cross section is much smaller and the nuclei are prevented from decaying. The transition between these two regimes occurs at an energy that is readily calculable and should be marked by feature in the interstellar spectrum of the ec nuclide. It was suggested that by identifying this feature in the spectrum measured near Earth it should be possible to determine the mean energy loss experienced by the cosmic rays in penetrating to the inner heliosphere [39]. Using the ec nuclides ${ }^{49} \mathrm{~V}$ and ${ }^{51} \mathrm{Cr}$ it was found that the difference between the energy loss experienced near solar maximum and that at solar minimum amounts to $\sim 200-300 \mathrm{MeV} /$ nuc [41] for element heavier than $\mathrm{H}$.

\section{CURRENT PICTURE}

Efforts to construct a consistent model of the origin of GCRs taking into account the various results cited above as well as observations of ultraheavy cosmic rays $[5,42]$ have led to the following picture $[43,44,45]$. Cosmic rays are thought to be accelerated in superbubbles, low density regions of the interstellar medium that contain contributions from relatively recent supernova explosions of many massive stars. Supernova shocks propagating through a superbubble accelerate both ions and charged dust grains, with approximately $20 \%$ of the accelerated matter coming from WR and other massive star contributions and the rest from old interstellar matter with a solar-like composition. For several nuclides, most notably ${ }^{22} \mathrm{Ne}$, the abundances in the massive star ejecta are sufficiently different from solar to be discernible after the mixing. Even the massive star ejecta have had at least $\sim 10^{5} \mathrm{yr}$ to mix with the ambient interstellar matter and during this time the primary ${ }^{59} \mathrm{Ni}$ has undergone electron capture decay.

Observational and modeling work in the coming years should further test the validity of this model. Likely areas for progress include the elemental composition of ultraheavy cosmic rays and the abundances of rare primary nuclides.

\section{REFERENCES}

1. P. Freier et al., Phys. Rev. 74, 1818 (1948).

2. E. P. Ney, "The Discovery of Heavy Cosmic Ray Nuclei" in Cosmic Abundances of Matter, edited by C. J. Waddington, AIP Conference Proceedings 183, Melville, New York, 1989, p. 366. 
3. P. S. Freier, "1948 Revisited" in Cosmic Abundances of Matter, edited by C. J. Waddington, AIP Conference Proceedings 183, Melville, New York, 1989, p. 377.

4. C. J. Waddington (2012), this volume.

5. M. H. Israel (2012), this volume.

6. P. Meyer, Ann. Rev. Astron. \& Astroph. 7, 1 (1969).

7. M. Bouffard et al., Astroph. \& Space Sci. 84, 3 (1982).

8. J. J. Engelmann et al., Astron. \& Astroph. 233, 96 (1990).

9. W. E. Althouse et al., IEEE Trans. Geosci. Electr. GE-16, 204 (1978).

10. D. E. Greiner, F. S. Bieser, \& H. H. Heckman, IEEE Trans. Geosci. Electr. GE-16, 163 (1978).

11. J. A. Simpson et al., Astron. \& Astroph. Suppl. Ser. 92, 365 (1992).

12. E. C. Stone et al., Space Sci. Rev. 86, 285 (1998).

13. H. L. Bradt \& B. Peters, Phys. Rev. 80, 943 (1950).

14. S. Hayakawa, K. Ito, \& Y. Terashima, Prog. Theor. Phys. Suppl., 8, No. 6, 1 (1958).

15. J. A. Simpson, Ann. Rev. Nucl. Part. Sci. 33, 323 (1983).

16. A. W. Strong, I. V. Moskalenko, \& V. S. Ptuskin, Ann. Rev. Nucl. Part. Sci. 57, 285 (2007).

17. J. S. George et al., Astroph. J. 698, 1666 (2009).

18. M. Ave et al., Astroph. J. 697, 106 (2009).

19. M. Garcia-Munoz, G. M. Mason, \& J. A. Simpson, Astroph. J. Lett. 201, L141 (1975).

20. M. E. Wiedenbeck \& D. E. Greiner, Astroph. J. Lett. 239, L139 (1980).

21. J. A. Simpson \& J. J. Connell, Space Sci. Rev. 97, 337 (2001).

22. N. E. Yanasak et al., Astroph. J. 563, 768 (2001).

23. M. A. DuVernois \& M. R. Thayer, Astroph. J. 465, 982 (1996).

24. M. E. Wiedenbeck et al., Space Sci. Rev. 99, 15 (2001).

25. M. E. Wiedenbeck et al., Space Sci. Rev. 130, 415 (2007).

26. M. Cassé \& P. Goret, Astroph. J. 221, 703 (1978).

27. J.-P. Meyer, Astroph. J. Suppl. 57, 173 (1985).

28. R. I. Epstein, Mon. Not. Royal Astron. Soc. 193, 723 (1980).

29. C. J. Cesarksy \& J.-P. Bibring, "Cosmic-ray Injection into Shock Waves" in Origin of Cosmic Rays, edited by G. Setti et al., IAU Symposium 94, 1981, p. 361.

30. J.-P. Meyer, L. O'C. Drury, \& D. C. Ellison, Astroph. J. 487, 182 (1997).

31. D. C. Ellison, L. O'C. Drury, \& J.-P. Meyer, Astroph. J. 487, 197 (1997).

32. R. C. Maehl et al., Astroph. J. Lett. 202, L119 (1975).

33. M. Garcia-Munoz, J. A. Simpson, \& J. P. Wefer, Astroph. J. Lett. 232, L95 (1979).

34. R. A. Mewaldt et al., Astroph. J. Lett. 235, L95 (1980).

35. M. Cassé \& J. A. Paul, Astroph. J. 258, 860 (1982).

36. N. Prantzos, Adv. Space Res. 4, 109 (1984).

37. W. R. Binns et al., Astroph. J. 634, 351 (2005).

38. A. Soutoul, M. Cassé, \& E. Juliusson, Astroph. J. 219, 753 (1978).

39. G. M. Raisbeck et al., Proc. 14th Int. Cosmic Ray Conf. (Munich) 3, 937 (1975).

40. M. E. Wiedenbeck et al., Astroph. J. Lett. 523, L61 (1999).

41. S. M. Niebur et al., J. Geophys. Res. 108, 8033 (2003).

42. B. F. Rauch et al., Astroph. J. 697, 2083 (2009).

43. R. E. Lingenfelter \& J. C. Higdon, Astroph. J. 660, 330 (2007).

44. W. R. Binns et al., Space Sci. Rev. 130, 439 (2007).

45. R. E. Lingenfelter (2012), this volume. 